\title{
Production of (super)heavy quarkonia and new Higgs physics at hadron colliders
}

\author{
G.A. Kozlov, A.N. Sissakian, J.I. Khubua, G. Arabidze and G. Khoriauli \\ Joint Institute for Nuclear Research, \\ 141980 Dubna, Moscow Region, Russia \\ e-mail: kozlov@thsun1.jinr.ru \\ and \\ T. Morii \\ Div. of Sciences for Natural Environment, \\ Faculty of Human Development, \\ Kobe University, Kobe, Japan \\ e-mail: morii@kobe-u.ac.jp
}

\begin{abstract}
Based on the two Higgs doublet model, we study the effect of Higgsboson exchange on the (super)heavy quarkonium $\bar{Q} Q$, which induces a strong attractive force between a (super)heavy quark $Q$ and an antiquark $\bar{Q}$. An interesting application is the decay of (super)heavy quarkonia $\bar{Q} Q$ into a Higgs boson associated with gauge bosons. The criterion for making the $\bar{Q} Q$ bound state is studied. We also show that non-perturbative effects due to gluonic field fluctuations are rather small in such a heavy quark sector. Possible enhancement for productions and decays of $\bar{Q} Q$ bound states made from the fourth generation quark $Q$ is discussed for $\bar{p} p$ (at the Tevatron) and $p p$ (at the LHC) collisions.
\end{abstract}

PACS 12.38.Aw, 12.38.Lg, 12.40.Qq, 14.80.Dq, 14.80.Gt, 14.80.Er

\section{INTRODUCTION}

A study on quarkonia $T(\bar{Q} Q)$ composed of a (super)heavy quark $Q$ and an antiquark $\bar{Q}$ (a possible and interesting candidate of $Q$ is the $\operatorname{up}(U)$ - and/or $\operatorname{down}(D)$-quarks in the fourth generation family) is required in current particle physics for testing the standard model (SM) and/or searching for signals for physics beyond the SM. It is nowadays one of the most interesting subjects since the subject can be studied, with high priority, in the forthcoming experiments at high energy hadron colliders, i.e. the Tevatron and LHC. In particular, no theoretical arguments are seen to rule out the (super)heavy quarks and the (super)heavy quarkonium states with the masses around hundred $\mathrm{GeV}$ or even a few $\mathrm{TeV}$ (see, e.g. [1]). As the recent example, in one of the extended models, the little Higgs model [2], there could be an additional heavy quark $\tilde{q}$ with a mass of order $\mathrm{O}(1 \mathrm{TeV})$ to promote the quark doublet $q$ to quark triplet under the global $\mathrm{SU}(3), Q=(q, \tilde{q})$. By preserving the global symmetry of the coupling, the one-loop quadratic divergence to the top quark is removed. In fact, the SM and its extensions, e.g., the Minimal Supersymmetric Standard Model (MSSM), do not explain the family (generation) structure of the quark masses. Each quark has an arbitrary Yukawa coupling and hence is independent of the family to which it belongs. It is required to explain the family structure and the Cabibbo-Kobayashi-Maskawa (CKM) matrix for the quark sector in any extension of the SM or even in the SM. 
It is known from the history of particle physics that the first signals for $c$ - and $b$-quarks in hadronic collisions were leptonic decays of their $J / \psi(\bar{c} c)$ or $\Upsilon(\bar{b} b)$ bound states. Can (super)heavy quarks also be first discovered through the decay of their quark-antiquark bound states into lepton pairs? It seems the answer is apparently not transparent, because one of the main properties of (super)heavy quarkonia is concerned with the appearance of new decay modes into weak bosons and even Higgs bosons in the final states. Once the Higgs boson $H$ is discovered, one needs to measure its couplings to other particles. The value of $H$-boson couplings can be extracted by measuring a variety of Higgs boson productions and their decay modes. Thus, it is important to find the $H$-boson in as many channels as possible, including its production coming from the decay of (super)heavy quarkonia. Such a Higgs boson can be looked for in the following new decay modes; $T(\bar{Q} Q) \rightarrow H Z$, $T\left(\bar{Q}_{1} Q_{1}\right) \rightarrow H T\left(\bar{Q}_{2} Q_{2}\right), T(\bar{Q} Q) \rightarrow \gamma H$ and $T(\bar{Q} Q) \rightarrow g g H, \gamma \gamma H$, where $T(\bar{Q} Q)$ carries quantum numbers of $J^{P C}=1^{--}, 0^{-+}$. Production of heavy quarkonia like $\bar{b} b$ and $\bar{t} t$ associated with a Higgs boson emission in the decay of extra gauge bosons $Z^{\prime}$ has been studied in $[3,4]$. Here we are interested in the case in which the new decay modes mentioned above become dominant and hence the branching ratio for a single heavy quark decay accompanying the real weak boson emission $Q \rightarrow q W$ ( $q$ is a lighter quark), leaving $q$ as a spectator, is small. Of course, it is necessary to examine whether the spectator mode can be dominant or not.

The direct decay of the top quark, $t \rightarrow W b[5,6]$, and the flavor changing top quark decays, $t \rightarrow c \gamma(c g, c Z)$ and $t \rightarrow c H$ [7], in the both SM and two-Higgs doublet model (2HDM), have been studied intensively for the last decade. The SM predictions of the branching ratios $(B R)$ for those decays $t \rightarrow c \gamma, t \rightarrow c g, t \rightarrow c Z$ and $t \rightarrow c H$ are significantly small, being $B R \sim 5 \times 10^{-13}, 4 \times 10^{-11}, 1.3 \times 10^{-13}$ and $10^{-14}-10^{-13}$, respectively. Obviously, the rare decays are out of interest here since they are very difficult to be observed in hadron colliders even at the highest luminosity and thus we neglect them in this work. The sensitivity of current experiments at Tevatron Run II or future experiments at forthcoming LHC closely approaches the rate required for ruling out the Higgs boson production or discovering it through the decay of (super)heavy quarkonia mentioned above. In this connection, precise theoretical estimates of the rates are required for an unambiguous interpretation of experimental upper limits.

One cannot exclude the possibility of the new strong interactions which primarily control the dynamics of (super)heavy quarks such as the 4 th generation $\operatorname{up}(U)$ and/or $\operatorname{down}(D)$ quarks. In one of the "top-color" models [8] with $1 \mathrm{TeV}$ scale, there is the following "topcolor" gauge structure

$$
\begin{aligned}
S U(3)_{4} \times S U(3)_{h} \times S U(3)_{l} \times U(1)_{Y_{4}} \times U(1)_{Y_{h}} \times U(1)_{Y_{l}} \times S U(2)_{L} \\
\rightarrow S U(3)_{Q C D} \times U(1)_{E M},
\end{aligned}
$$

where $S U(3)_{4} \times U(1)_{Y_{4}}, S U(3)_{h} \times U(1)_{Y_{h}}$ and $S U(3)_{l} \times U(1)_{Y_{l}}$ generally coupled to the 4th, 3rd and first two generations, respectively. The $U(1)_{Y_{i}}$ are just rescaled versions of electroweak $U(1)_{Y}$ into the strongly interacting world. In this model, below the symmetrybreaking scale $\mu_{S B}$, the spectrum includes massive "top-gluons", which mediate vectorial color-octet interactions among (super)heavy quarks $Q(=t, U, D)$

$$
-\left(4 \pi \kappa / \mu_{S B}^{2}\right)\left(\bar{Q} \gamma_{\mu} \frac{\lambda^{a}}{2} Q\right)^{2}
$$


If the coupling $\kappa$ lies above some critical value $\kappa_{\text {crit }}$, the heavy quark condensate $\langle\bar{Q} Q\rangle$ can be formed. The strong "top-color" dynamics can bind $\bar{Q}$ and $Q$ into a set of "heavy-pions" $(\bar{Q} Q)$. The criterion for existence of a (super)heavy quarkonium is that the binding energy $\epsilon_{B}$ should be larger at least than the total decay width $\Gamma_{\text {tot }}$ of its quarkonium, namely $c=\left(\Gamma_{t o t} / \epsilon_{B}\right)<1$. Since such a (super)heavy quark-antiquark bound state is considered to be a non-relativistic system, the quark potential model should be applicable to the analysis. Then as pointed out in [9-11], one cannot exclude the possibility of the Higgs-boson interaction which dominates significantly over the one-gluon exchange $\sim-(4 / 3) \alpha_{s}\left(m_{Q}\right) / r$ for the (super)heavy quarkonium, where $\alpha_{s}$ is the strong coupling constant depending on the quark mass $m_{Q}$ and $r$ is a distance between a quark and an antiquark. We show in Sec.2 that the strong binding force due to the Higgs-boson exchange gives rise to a necessary condition $c<1$ for enabling the heavy quarkonium to exist and leading to observation of its resonance.

On the other hand, in the physics of interplay among quarks, it is well known that the exact QCD vacuum should contain the fluctuations of gluonic fields at large scales [12]. Those non-perturbative fluctuations cause the distortion of interactions between quarks and antiquarks. We consider (super)heavy quarks as external objects allocated in the gluonic vacuum. Here, we study those non-perturbative fluctuation effects of the gluon field on the decay of a quarkonium $T(\bar{U} U)$ into the Higgs- and $Z$-bosons. Our result is based on the wellknown statement (see, e.g., [12]) that the non-perturbative effect on dynamics of heavy quark systems is expressed in terms of vacuum expectation values of the local operators constructed from gluonic field operators. The leading effect is proportional to a matrix element of the form $\left\langle G^{a}{ }_{\mu \nu}{ }^{2}(0)\right\rangle_{0}$, where $G_{\mu \nu}^{a}(x)$ is the standard gluonic field strength tensor with color indices $a=1,2, \cdots, 8$. The lowest level of the (super)heavy quarkonium is determined by the color-singlet Yukawa-type attractive force mediated by the "light" scalar $\chi$-boson. Here, we are interested in the corrections due to non-perturbative gluonic fluctuations in the exact QCD vacuum. In this paper, we show that in a (super)heavy quarkonium decay such as $T(\bar{U} U) \rightarrow h Z$ ( $h$ means a lightest CP-even Higgs-boson in 2HDM), the non-perturbative fluctuation effect of the gluonic field can be calculated, to some extent, without detailed knowledge of the vacuum structure and furthermore, it gives a negligible result.

The outline of this work is organized as follows. In Sec. 2, we discuss an effective potential mediated by a Higgs boson. An effective model for the lower-energy theorem will be discussed in Sec. 3. Finally, in Sec. 4, we give our conclusion and discussion.

\section{EFFECTIVE POTENTIAL VIA HIGGS BOSON EXCHANGE}

First of all, one should notice that the total cross-section for the process $p p(\bar{p} p) \rightarrow \bar{Q} Q$ is strongly related to the underlying subprocesses. For example, at $\sqrt{s}=14 \mathrm{TeV}$, the cross section for various processes of $Q$ and $\bar{Q}$ pair productions with $Q=U$ and/or $D$ in the mass region $m_{U} \simeq m_{D} \simeq m_{4}=0.2-0.6 \mathrm{TeV}\left(m_{4}\right.$ is the mass of the fourth generation quark), becomes $\sigma \simeq 10^{-1}-10^{-2}(\mathrm{pb})$ and $\sigma \simeq 1-10^{-2}(\mathrm{pb})$ for $\gamma / Z$ production and $W$ production, respectively, in the $q$ and $\bar{q}$ annihilation channels, and $\sigma \simeq 10-10^{-1}$ (pb) for the two-gluon fusion channel.

Let us consider a production (in $p p$ or $\bar{p} p$ ) of (super)heavy quarkonium $T(\bar{Q} Q$ ) followed by the decay process $T(\bar{Q} Q) \rightarrow h Z$ with $Q=t, U, D$, being assumed to be the dominant decay process of $T(\bar{Q} Q)$. Then, it is supposed that this dominant mechanism at high transverse 
momentum involves, e.g. the production of a gluon that produces a color-octet $\bar{Q}$ and $Q$ pair which then fragments into a color-singlet bound state $T(\bar{Q} Q)$ by emitting two or more soft gluons. This $T(\bar{Q} Q)$-state should be transversely polarized at high transverse momentum, since it is emanated from a gluon which has only transverse polarization states.

In the lowest bound state $\bar{Q} Q$, the quark $Q$ and the antiquark $\bar{Q}$ are assumed to be located at a distance

$$
r \sim\left[m_{Q} \lambda\left(m_{Q}\right)\right]^{-1}
$$

which is small compared to the scale of strong interactions. $\lambda\left(m_{Q}\right)$ is a strength of the interaction between a quark and an antiquark. The wavefunction of the lowest bound state is proportional to $\exp (-\mu r)$ with $\mu \sim m_{Q} \lambda\left(m_{Q}\right)$. Note that for $r_{0}=\mu^{-1}$ being smaller than in the typical size of fluctuations, our approach becomes applicable since the potential dumps exponentially for a distances $r \gg \mu^{-1}$.

Let us consider a simple model (Model I) where the dominant effective potential for a $\bar{Q}$ and $Q$ system at a small distance looks like $[10,11]$

$$
V_{e f f}(r) \sim-\frac{C_{F}}{r} \alpha_{s}\left(m_{Q}\right)-\frac{\lambda\left(m_{Q}, \xi_{\chi Q}\right)}{r} \exp \left(-m_{\chi} r\right)
$$

with

$$
\lambda\left(m_{Q}, \xi_{\chi Q}\right)=\frac{m_{Q}^{2}}{4 \pi v^{2}} \xi_{\chi Q}^{2}
$$

and $\xi_{\chi Q}$ reflects the model "flavor" in the strength of the interaction between the scalar $\chi$-boson and a heavy quark $Q\left(\xi_{\chi Q}=1\right.$ in the minimal SM, otherwise $\left.\xi_{\chi Q}>1\right)$. $v$ is the vacuum expectation value of Higgs boson, $v=246 \mathrm{GeV}$ (in $2 \mathrm{HDM}, v=\sqrt{v_{1}^{2}+v_{2}^{2}}, v_{1}$ and $v_{2}$ are two neutral Higgs field vacuum expectation values) and $C_{F}$ is the color factor, $C_{F}=4 / 3$ for color $S U(3)$ group. In Fig.1, we show the ratio of the combined coupling $\alpha_{\text {comb }}=(4 / 3) \alpha_{s}\left(m_{Q}\right)+\lambda\left(m_{Q}, \xi_{\chi Q}\right) \cdot \exp \left(-m_{\chi} r\right)$ (see $\left.(4)\right)$ to the pure QCD coupling $\bar{\alpha}_{s}=$ $(4 / 3) \alpha_{s}$, as a function of a heavy quark mass $m_{Q}$ for different values of $\xi_{\chi Q}$ and $m_{\chi}$. The second term in $\alpha_{c o m b}$ is appropriate for $r \sim\left[m_{Q} \lambda\left(m_{Q}\right)\right]^{-1}$. The ratio becomes somewhat bigger for smaller Higgs-boson masses.

Because of our demand, $\lambda\left(m_{Q}, \xi_{Q \chi}\right)>C_{F} \alpha_{s}\left(m_{Q}\right)$, for a relevance of the $\chi$-boson interaction, the lower bound on $m_{Q}$ is given as

$$
m_{Q}>\frac{v}{\xi_{\chi Q}}\left(4 \pi C_{F} \alpha_{s}\right)^{1 / 2}
$$

which leads to $m_{Q} \geq m_{t}$ even if $\xi_{\chi Q}=2$ (see Fig.2). 

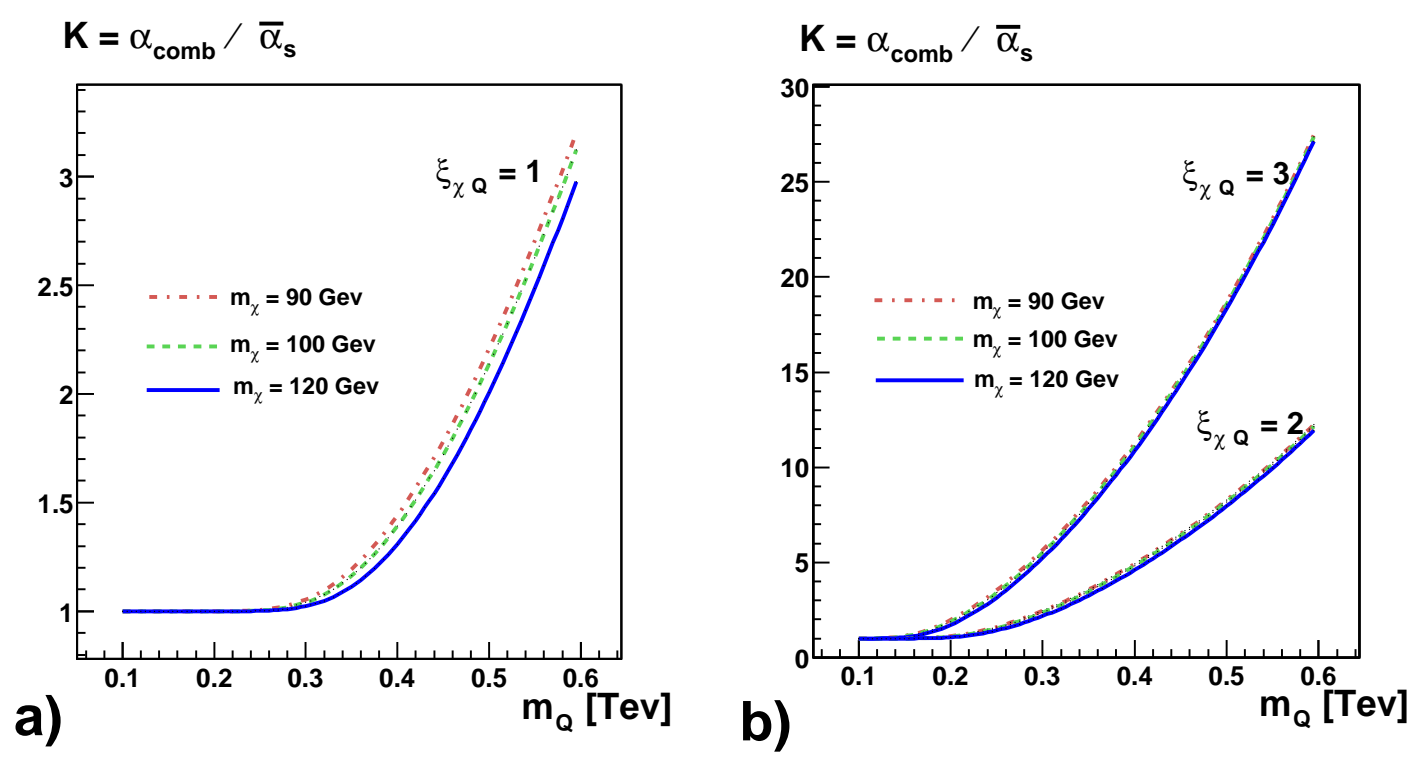

Fig. 1 Ratio of the combined coupling $\alpha_{c o m b}$ to the pure QCD coupling $\bar{\alpha}_{s}$ as a function of a heavy quark mass for (a) $\xi_{\chi Q}=1$ and (b) $\xi_{\chi Q}=2,3$. The curves are presented for $\chi$-boson masses $m_{\chi}=90,100$ and $120 \mathrm{GeV}$.

The requirement of the positivity of the variational parameter

$$
\mu \simeq \frac{\lambda m_{Q}}{2} \frac{\left(\lambda m_{Q}\right)^{2}-m_{\chi}^{2}}{\left(\lambda m_{Q}\right)^{2}+2 m_{\chi}^{2}}
$$

entered in both the bound state wavefunction $\Psi(r)=2 \mu^{-3 / 2} \exp (-\mu r)$ and the binding energy (see, for details, [11]),

$$
\epsilon_{B}=2 \lambda \frac{\mu^{3}\left(2 \mu-m_{\chi}\right)}{\left(2 \mu+m_{\chi}\right)^{3}}
$$

leads to an upper limit on $m_{\chi}$ (see Fig.3).

In the Model $\mathrm{I}$, the ratio $c=\left(\Gamma_{t o t} / \epsilon_{B}\right)<1$ could be guaranteed for $T(\bar{U} U)$ quarkonia to be formed by a strong attractive force via scalar Higgs-boson exchange with a sufficiently "hard" Yukawa coupling $\lambda\left(m_{Q}, \xi_{\chi Q}\right)$. The total decay width $\Gamma_{\text {tot }}$ is given by a sum of two terms

$$
\Gamma_{t o t}=\Gamma_{T}+\Gamma_{U}
$$

where the width $\Gamma_{T}$ is defined by the following decay channels

$$
T(\bar{U} U) \rightarrow h Z, \gamma Z, \gamma h, W^{+} W^{-}, \bar{b} b, \bar{t} t, \tau^{+} \tau^{-}, \mu^{+} \mu^{-}, g g g
$$




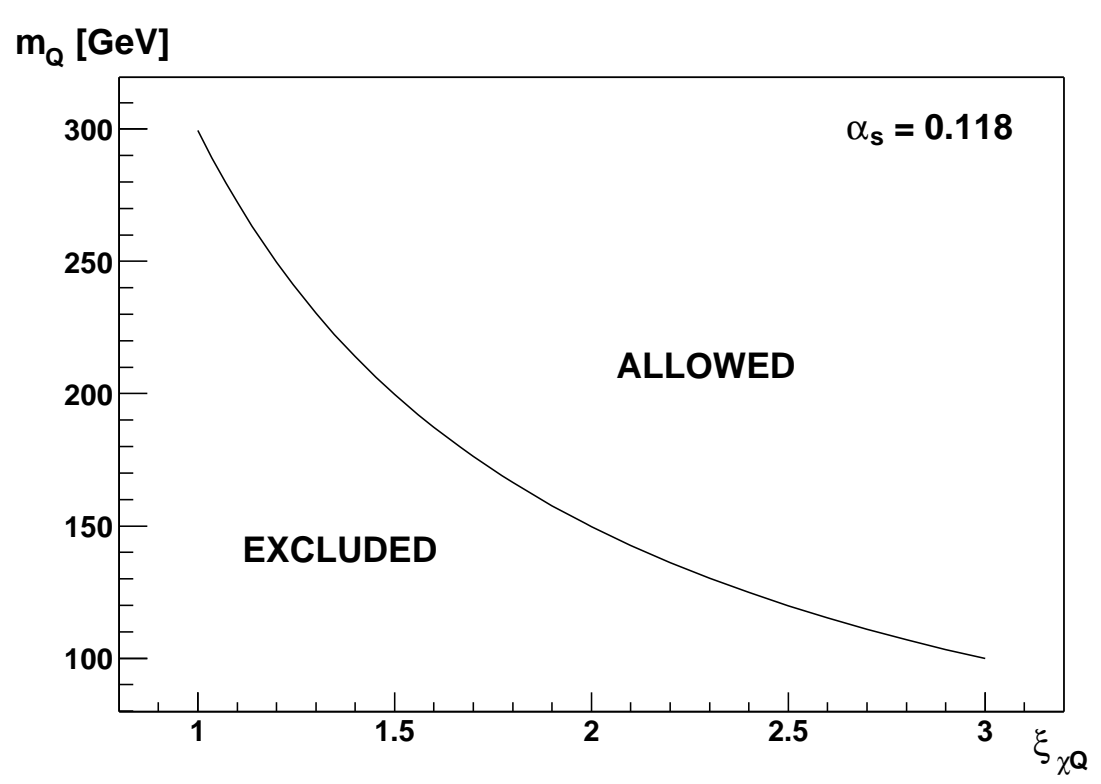

Fig.2 Lower bound on (super)heavy quark masses as a function of $\xi_{\chi Q}$.

and the single quark decay width $\Gamma_{U}$ is a sum of the following contributions: $U \rightarrow$ $D W^{+}, b W^{+}, b H^{+}\left(H^{+}\right.$is the charged Higgs-boson in $\left.2 \mathrm{HDM}\right)$. In the decays of $T(\bar{U} U)$ presented above, the radiative channel is suppressed by the coupling constant $\alpha$, the $g g g$ channel gives the small contribution due to $\alpha_{s}^{3}$ factor, and furthermore, the productions of the pairs of quarks and antiquarks or leptons and antileptons are also small because they follow via twoloop diagrams, where the amplitude is very small because of the presence of $\alpha$ (intermediate photons) or the Fermi constant $G_{F}$ (virtual $W^{ \pm}$-bosons).

We do not consider the contributions from the decay $U \rightarrow b H^{+}$because it is expected to have a rather small probability to be observed as expected from the following consideration: the Higgs-boson mass sum rule

$$
m_{H^{+}}^{2} \simeq\left(m_{A}^{2}+m_{W}^{2}\right)(1+\delta)
$$

with one-loop correction $\delta<10 \%$ [13] does not allow the production of charged Higgsbosons in the decay of top quark and, perhaps, also of $U$ quark due to the kinematical reason in the decoupling limit, $\left(m_{W}^{2} / m_{A}^{2}\right) \ll 1\left(m_{A}\right.$ is the CP-odd Higgs-boson in 2HDM). In addition, the experimental data at the Tevatron do not yet clarify the status of $t \rightarrow H^{+} b$ decay. The CDF results in direct search of a $\tau$-lepton emission from top quark decays give an upper limit on the branching ratio $B R\left(t \rightarrow H^{+} b\right) \sim 0.5-0.6$ at 95\% C.L. in the range $60 \mathrm{GeV}<m_{H^{+}}<160 \mathrm{GeV}$, assuming $B R\left(H^{+} \rightarrow \tau \nu_{\tau}\right)=1$ [14]. Furthermore, the D0 Collaboration excludes $B R\left(t \rightarrow H^{+} b\right)>0.36$ at $95 \%$ C.L. in the region $0.3<\tan \beta<150$ and $m_{H^{+}}<160 \mathrm{GeV}$ [15]. Assuming $\sum_{X=H^{+}, W^{+}} B R(t \rightarrow b X)=1$, the decay width of the $H^{+}$-boson channel in $U$ - and $t$-quark decays is small and hence this channel is out of interest.

Therefore, for the case of $U$ quarks, one can expect that the $\Gamma_{t o t}$ is given by

$$
\Gamma_{t o t}=\Gamma_{T}\left(T(\bar{U} U) \rightarrow h Z, W^{+} W^{-}\right)+\Gamma_{U}\left(U \rightarrow D W^{+}, b W^{+}\right) .
$$




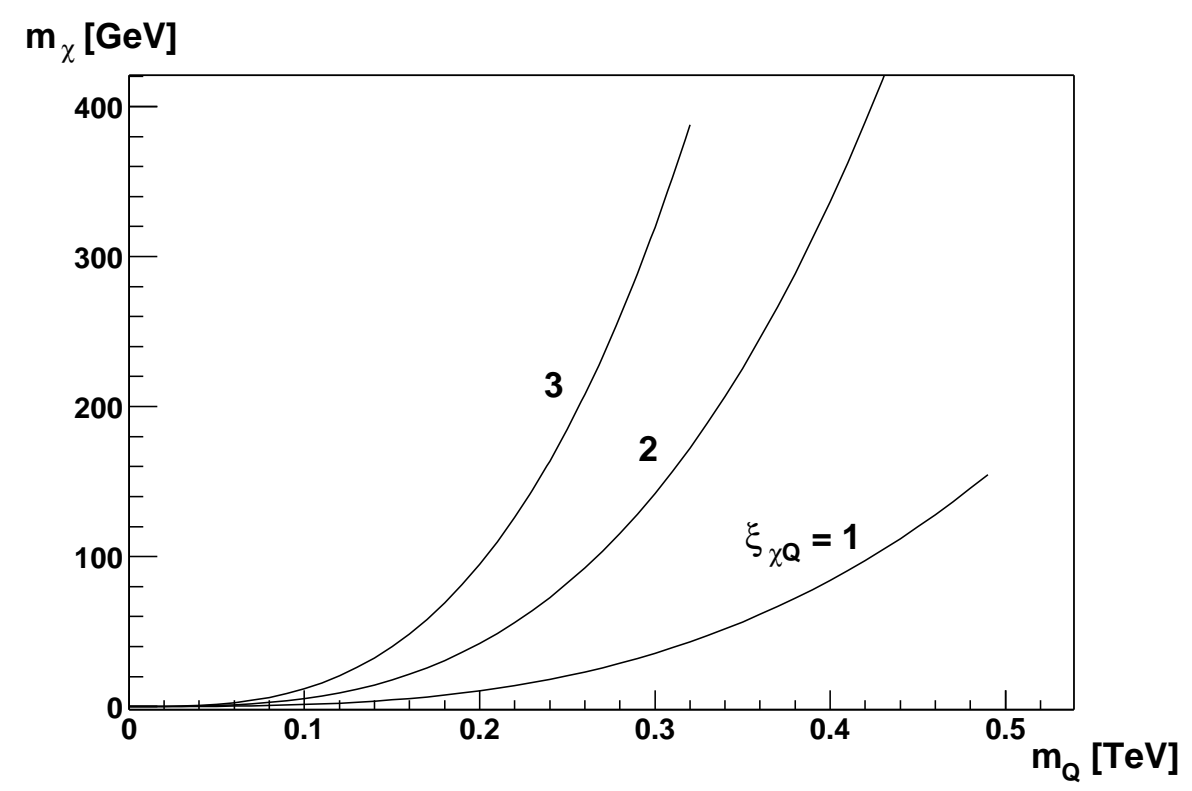

Fig.3 Upper limit on the scalar $\chi$-boson mass as a function of $m_{Q}$ for different values of $\xi_{\chi Q}$. The regions above the corresponding curves are excluded.

The main contribution to $\Gamma_{T}$ arises from the channel $T(\bar{U} U) \rightarrow h Z$, whose decay width for $T(\bar{U} U)\left(1^{--}\right)$is given by the following expression (see, e.g., [11])

$$
\Gamma(T(\bar{U} U) \rightarrow h Z)=\frac{\lambda^{3} m_{U}}{16} \eta_{h U}^{2}\left[\lambda \alpha_{z} v_{U}^{2}+\frac{1}{2} \alpha_{W}^{2}\left(\frac{m_{U}}{m_{W}}\right)^{4}\right] f^{3} \Phi,
$$

with

$$
\begin{gathered}
\alpha_{W}=\alpha / s_{W}^{2}, \alpha_{z}=\alpha_{W} / c_{W}^{2}\left(s_{W} \equiv \sin \theta_{W}, c_{W} \equiv \cos \theta_{W}\right) \\
v_{U}=\left(1-\frac{8}{3} s_{W}^{2}\right), \Phi=\left(1-\frac{4 m_{h}^{2}}{M_{T}^{2}}\right)^{1 / 2} \\
f \equiv f\left(\lambda, m_{U}, m_{\chi}\right)=\frac{\left(\lambda m_{U}\right)^{2}-m_{\chi}^{2}}{\left(\lambda m_{U}\right)^{2}+2 m_{\chi}^{2}}, M_{T} \simeq 2 m_{U} .
\end{gathered}
$$

$m_{U}$ is the mass of an $u p(U)$-quark of the fourth generation. We suppose that the couplings of the Higgs-boson $h$ and the $U$-quark have the same form as those for the couplings of the Higgs-boson $h$ and the top-quark (see the review [16])

$$
\eta_{h U} \simeq 1+\frac{m_{Z}^{2}}{m_{A}^{2}} s_{2 \beta} c_{2 \beta} \tan ^{-1} \beta
$$

where $s_{2 \beta}\left(c_{2 \beta}\right) \equiv \sin 2 \beta(\cos 2 \beta)$ and $\tan \beta$ is the standard ratio between two vacuum expectation values for two Higgs doublets in $2 \mathrm{HDM}$.

Using the standard formulae [5,6], the single $U$-quark decays are given for $U \rightarrow D W^{+}$ channel by

$$
\Gamma_{U}\left(U \rightarrow D W^{+}\right)=\frac{G_{F} m_{U}^{3}}{8 \sqrt{2} \pi}\left|V_{U D}\right|^{2}\left[\left(1-\frac{m_{D}^{2}}{m_{U}^{2}}-\frac{m_{W}^{2}}{m_{U}^{2}}\right)^{2}-4 \frac{m_{D}^{2} m_{W}^{2}}{m_{U}^{4}}\right]^{1 / 2}
$$




$$
\times\left[\left(1-\frac{m_{D}^{2}}{m_{U}^{2}}\right)^{2}+\left(1+\frac{m_{D}^{2}}{m_{U}^{2}}\right) \cdot \frac{m_{W}^{2}}{m_{U}^{2}}-2 \frac{m_{W}^{4}}{m_{U}^{4}}\right]
$$

and for $U \rightarrow b W^{+}$decay by

$$
\Gamma_{U}\left(U \rightarrow b W^{+}\right)=\Gamma_{0}\left(U \rightarrow b W^{+}\right)(1-\Delta)
$$

where

$$
\Gamma_{0}\left(U \rightarrow b W^{+}\right)=\frac{G_{F} m_{U}^{3}}{8 \sqrt{2} \pi}\left|V_{U b}\right|^{2} \beta_{W}^{4}\left(3-2 \beta_{W}^{2}\right)
$$

with $\beta_{W}=\sqrt{1-m_{W}^{2} / m_{U}^{2}}$. $V_{U D}$ and $V_{U b}$ are generalized CKM matrix elements and the $O\left(\alpha_{s}\right)$ QCD correction [6] in (15) is

$$
\Delta=\frac{C_{F} \alpha_{s}}{2 \pi}\left(\frac{2 \pi^{2}}{3}-\frac{5}{2}\right) .
$$

As an example, for a typical set of parameters: $m_{U}=400 \mathrm{GeV}, m_{D}=300 \mathrm{GeV}, m_{\chi} \simeq m_{h}=$ $100 \mathrm{GeV},\left|V_{U D}\right| \simeq 1, \xi_{\chi U}=2\left(\eta_{h U} \simeq 1\right)$ we give the numerical results for those decay widths

$$
\begin{aligned}
\Gamma(T(\bar{U} U) \rightarrow h Z) & \simeq 2.2 \eta_{h U}^{2} \mathrm{GeV} \\
\Gamma\left(U \rightarrow D W^{+}\right) & \simeq 1.21 \mathrm{GeV}, \quad \Gamma\left(U \rightarrow b W^{+}\right) \simeq 0.0021 \mathrm{GeV},
\end{aligned}
$$

where $c=\Gamma_{\text {tot }} / \epsilon_{B}=0.27$ at the calculated value $\epsilon_{B}=12.65 \mathrm{GeV}$.

The contribution of the decay $U \rightarrow b W^{+}$is negligible due to rather small value of $\left|V_{U b}\right| \sim 10^{-2}$. We have checked the possibility of the existence of bound states $T(\bar{Q} Q)$ composed of $U$ (and $D$ )-quarks, starting at the lowest value of $\xi_{\chi Q} \geq 1$, where the increasing $\xi_{\chi Q}$ gives rise to an effect on $c^{-1}>1$ (see Fig.4).

The expected event topology of the decays $U \rightarrow b W^{+}$and $U \rightarrow D W^{+}$are similar to that of $t \rightarrow b W^{+}$. However, for the down-type heavy quark $D$, the dominant decay mode could be $D \rightarrow t W^{-}$and thus, in the case of $\bar{D} D$-pair production, the final state can consist of two pairs of leptons and neutrinos (originated from decays of the $t$ quark and $W$ boson) with different flavors, in general. Now, by taking, as an example, the following parameters: $m_{D}=$ $400 \mathrm{GeV}, m_{\chi} \simeq m_{h}=100 \mathrm{GeV}, \xi_{\chi D}=2,\left|V_{D t}\right| \simeq 0.012$, we can obtain the following decay widths

$$
\Gamma(T(\bar{D} D) \rightarrow h Z) \simeq 2.25 \eta_{h D}^{2} \mathrm{GeV}, \quad \Gamma(D \rightarrow t W) \simeq 1.47 \mathrm{MeV}
$$

One can see that the decay width $\Gamma(T(\bar{D} D) \rightarrow h Z)$ can be enhanced by the Yukawa-flavor factor (within $2 \mathrm{HDM}) \eta_{h D}=-\sin \alpha / \cos \beta$ at large values of $\tan \beta$.

For comparison, let us consider an instructive example, i.e. the decay of $T(\bar{U} U)\left(0^{-+}\right)$ states to $h$ - and $Z$-boson, where the decay width is given by the following expression (see, e.g., $[11])$ 


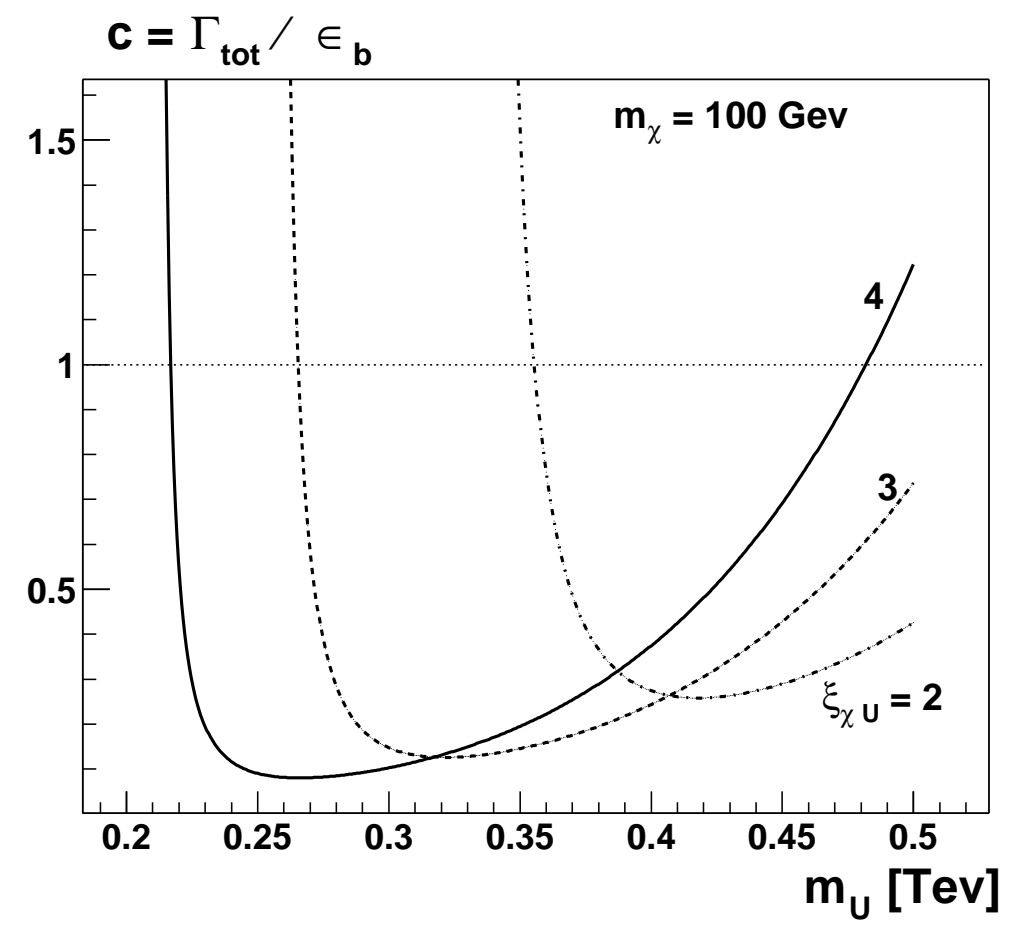

Fig.4 Ratio $c=\Gamma_{\text {tot }} / \epsilon_{B}$ being responsible for occurrence of the bound state $T(\bar{U} U)$ as a function of the quark mass $m_{U}$ at different values of $\xi_{\chi U}$ for $m_{\chi}=100 \mathrm{GeV}$. The region below the value $c=1$ is allowed.

$$
\Gamma(T(\bar{U} U) \rightarrow h Z)=\frac{3 \lambda^{3} m_{U}}{32} \eta_{h U}^{2} \alpha_{z}^{2}\left(\frac{m_{U}}{m_{Z}}\right)^{4} f^{3} \Phi .
$$

The numerical estimation gives $\Gamma(T(\bar{U} U) \rightarrow h Z) \simeq 6.80 \eta_{h U}^{2} \mathrm{GeV}$, which is roughly 3 times larger than that in the case of $T(\bar{U} U)\left(1^{--}\right) \rightarrow h Z$ decay mode given in (18) and hence leads to larger production rate of $T(\bar{U} U)\left(0^{-+}\right)$than $T(\bar{U} U)\left(1^{--}\right)$. This simple example confirms our belief that the most promising candidate of the (super)heavy quarkonium which could be searched at the LHC should be the pseudoscalar state $T(\bar{U} U)\left(0^{-+}\right)$.

It is interesting to estimate the effect of the scalar $\chi$-boson exchange on the $(\bar{Q} Q)$ production cross-section at different $m_{\chi}$ as a function of $m_{Q}$.

If the $Q$-quark is relatively long-lived, the peak of the cross-section at a $\bar{Q} Q$ resonance due to the one-gluon exchange is given by

$$
\sigma_{c} \sim \alpha_{s}^{3}\left(\frac{m_{Q}}{\Gamma_{Q}}\right) \frac{1}{s}
$$

for a given center-of-mass energy $s$. The cross-section (21) has a strong sensitivity to $\alpha_{s}$ and decreases sharply with increasing $m_{Q}$ because of the rapid grougth of $\Gamma_{Q}$ (see Eqs. (14) and (15)). To leading order, the scalar $\chi$-boson exchange effect is taken into account simply by making the replacement

$$
\alpha_{s} \rightarrow \alpha_{s}+\tilde{\alpha}\left(m_{Q}, m_{\chi}, \xi_{\chi Q}\right),
$$


where

$$
\tilde{\alpha}\left(m_{Q}, \xi_{\chi Q}\right)=\frac{3 m_{Q}^{2}}{16 \pi v^{2}} \xi_{\chi Q}^{2} \exp (-\epsilon)
$$

with $\epsilon=m_{\chi} /\left(m_{Q} \lambda\right) \ll 1$. Here, we see a simple increase of the coupling strength between $\bar{Q}$ and $Q$. We find a relative enhancement of the effective cross-section $\sigma_{\text {eff }}$

$$
\sigma_{e f f}=\sigma_{c}\left(1+3 \tilde{\alpha} \alpha_{s}^{-1}+3 \tilde{\alpha}^{2} \alpha_{s}^{-2}+\tilde{\alpha}^{3} \alpha_{s}^{-3}\right)
$$

due to the $\chi$-boson exchange effect. In Fig. 5 we show the ratio of the effective cross-section (24) to the cross-section $(21)$ as a function of $m_{Q}$. The curve in Fig. 6 is appropriate for a massless $\chi$ - boson.

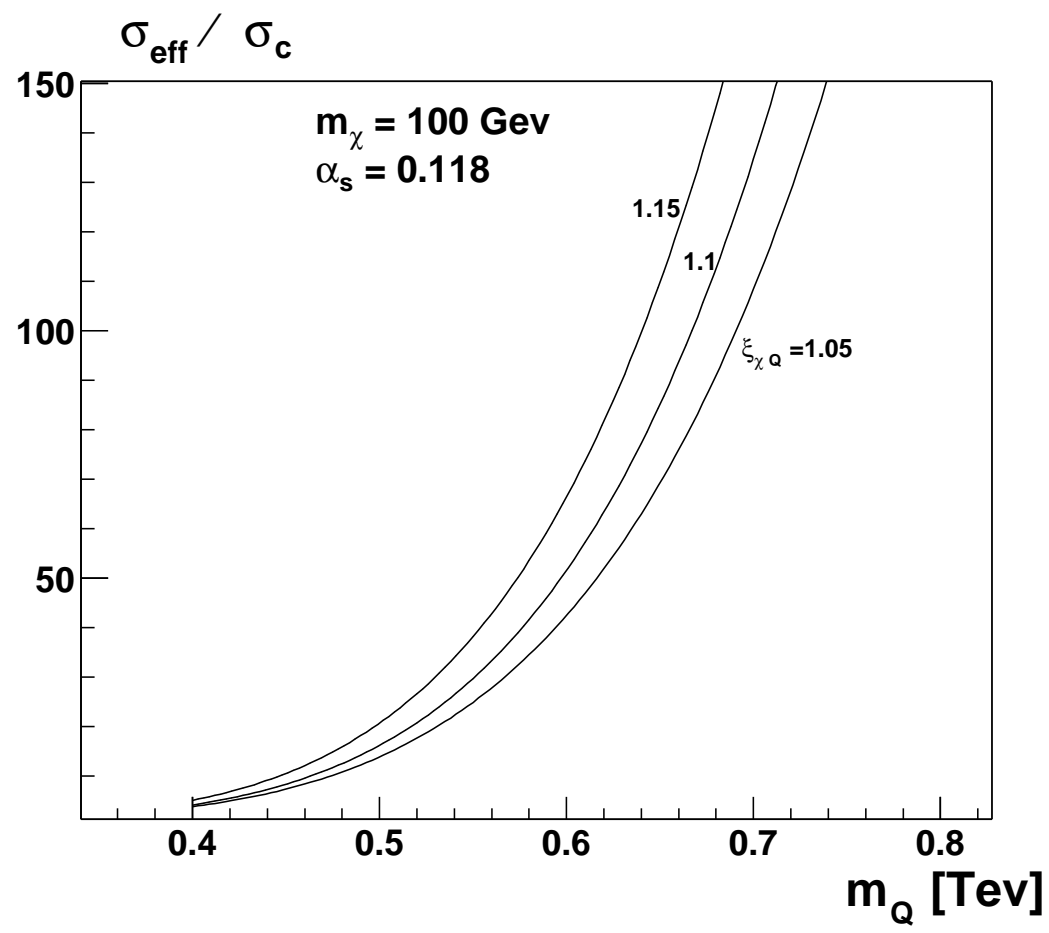

Fig.5 Ratio of the effective cross-section $\sigma_{\text {eff }}(24)$ and the cross-section $\sigma_{c}$ at the $1 \mathrm{~S}$ resonance $\bar{Q} Q$ as a function of $m_{Q}$. The curves are for $\xi_{\chi Q}=1.05,1.10,1.15$ and $m_{\chi}=100 \mathrm{GeV}$ at $\alpha_{s}=0.118$.

In the end of this Section we give briefly the results of the decays $\Phi \rightarrow T(\bar{Q} Q)+\gamma$ where a Higgs-boson $\Phi(\Phi=h$ or $\Phi=H)$ with 4-momentum $q_{\mu}$ and the mass $m_{\Phi}$ decays in the heavy vector $\left(1^{--}\right)$quarkonium $T(\bar{Q} Q)(Q=b$-quarks, $t$-quarks, ...), carrying the momentum $P_{\mu}=2 p_{\mu}\left(P^{2}=m_{T}^{2}\right)$, and a photon with the momentum squared $k^{2}=0$. 


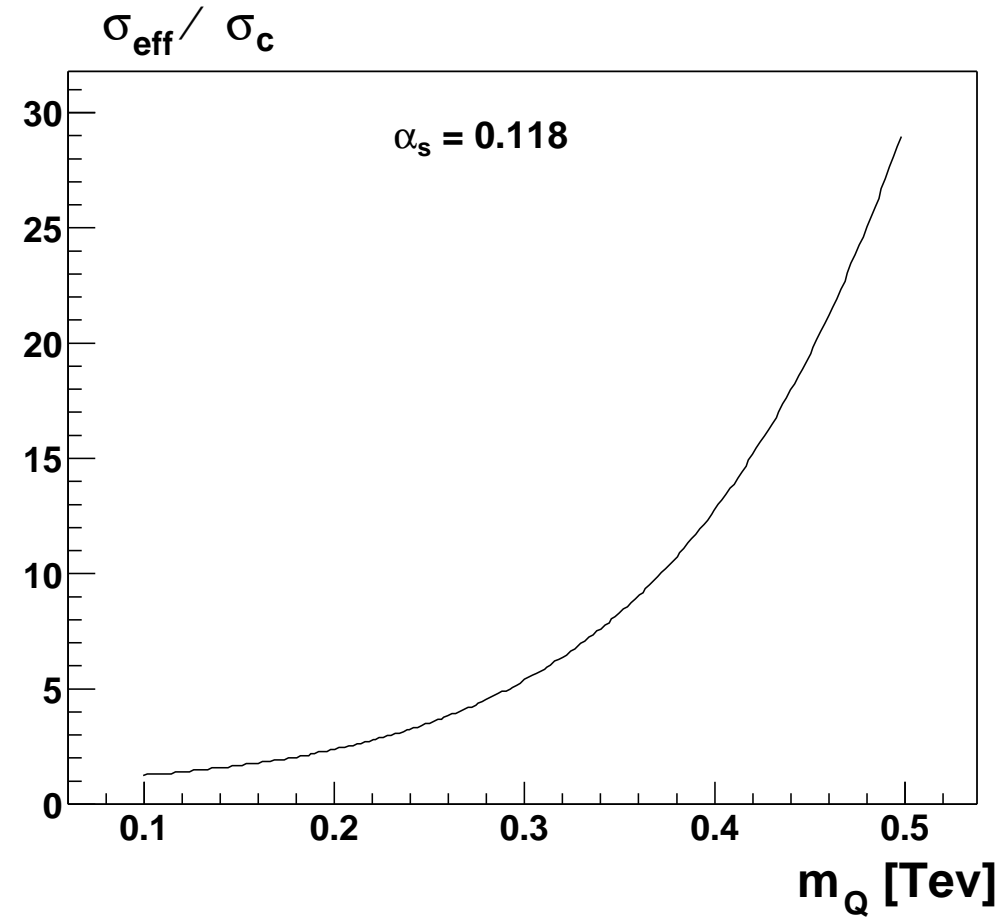

Fig.6 Ratio of the effective cross-section $\sigma_{\text {eff }}(24)$ and the cross-section $\sigma_{c}$ at the $1 \mathrm{~S}$ resonance $\bar{Q} Q$ as a function of $m_{Q}$. The curve is for the massless $\chi$-boson.

The amplitude of the transition $\Phi \rightarrow T \gamma$ is [17]

$$
A(\Phi \rightarrow T \gamma)=-2 \sqrt{4 \pi \alpha} g_{V} e_{Q} \frac{1}{v} \eta_{\Phi Q} \frac{1}{1-\left(m_{\Phi} / m_{Q}\right)^{2}} \epsilon^{\alpha} k^{\beta}\left(P_{\alpha} \phi_{\beta}-P_{\beta} \phi_{\alpha}\right),
$$

where $e_{Q}$ is the charge of the quark $Q, \phi_{\mu}$ being the polarization vector of $T(\bar{Q} Q)$ and $g_{V}$ is defined in the standard manner

$$
\left\langle T(\bar{Q} Q)\left|\bar{Q} \gamma_{\mu} Q\right| 0\right\rangle=m_{T}^{2} g_{V} \phi_{\mu}
$$

and can be estimated from the leptonic decay width $T(\bar{Q} Q) \rightarrow \overline{l l}(l=e, \mu, \tau)$ :

$$
\Gamma(T(\bar{Q} Q) \rightarrow \overline{l l})=\frac{4}{3} \pi\left(\alpha e_{Q} g_{V}\right)^{2} m_{T} .
$$

At an arbitrary large values of $y=\left(m_{\Phi} / 2 m_{Q}\right)^{2}>1$ there are the corrections to the amplitude (25) due to the one-loop $O\left(\alpha_{s}\right)$ gluon contributions

$$
1-F(y) C_{F} \alpha_{s}\left(m_{\Phi}^{2}-4 m_{Q}^{2}\right),
$$

where the function $F>0$ was calculated in Ref. [17]. For the decay processes $h \rightarrow \Upsilon(\bar{b} b) \gamma$, $H \rightarrow \Upsilon(\bar{b} b) \gamma$ and $H \rightarrow T(\bar{t} t) \gamma$ we are considered here as the promising channels in a wide range of the Higgs-boson mass we expect the relative decay width compared to final state quarks $\bar{Q} Q$, which is given by [17] 


$$
\begin{gathered}
f \equiv \frac{B R(\Phi \rightarrow T(\bar{Q} Q) \gamma)}{B R(\Phi \rightarrow \bar{Q} Q)}=\frac{\Gamma(\Phi \rightarrow T(\bar{Q} Q) \gamma)}{\Gamma(\Phi \rightarrow \bar{Q} Q)}= \\
\frac{64 \pi \alpha e_{Q}^{2}}{3} g_{V}^{2}\left(\frac{m_{Q}}{m_{\Phi}}\right)^{2} K^{2}\left(y, \alpha_{s}\right)\left[1-\left(\frac{m_{T}}{m_{\Phi}}\right)^{2}\right]^{-1 / 2}
\end{gathered}
$$

where

$$
K\left(y, \alpha_{s}\right) \simeq 1-\frac{\alpha_{s}\left(m_{\Phi}^{2}-m_{T}^{2}\right)}{\pi} C_{F} \ln 2 \ln (4 y)
$$

will be important for the processes $h \rightarrow \Upsilon(\bar{b} b) \gamma, H \rightarrow \Upsilon(\bar{b} b) \gamma$, where $\left(m_{b} / m_{\Phi}\right)^{2} \rightarrow 0$. In Figs. 7 and 8 we plot the relative widths of $h \rightarrow \Upsilon(\bar{b} b) \gamma$ and $H \rightarrow \Upsilon(\bar{b} b) \gamma$ decays, respectively, compared to $\bar{b} b$ final state, vs. $m_{h}$ and $m_{H}$, respectively, and $H \rightarrow T(\bar{t} t) \gamma$ decay (see Fig. 9) compared to $\bar{t} t$-state as a function of $m_{H}$.

The recognizing of the quark-antiquark bound state can be shown through the resonance structure having a specific signal, e.g., the final leptonic pairs $e^{+} e^{-}, \mu^{+} \mu^{-}$and $\tau^{+} \tau^{-}$. This resonance is expected can give one clear evidence of a bound state production over the QCD background if the production would be significantly large.

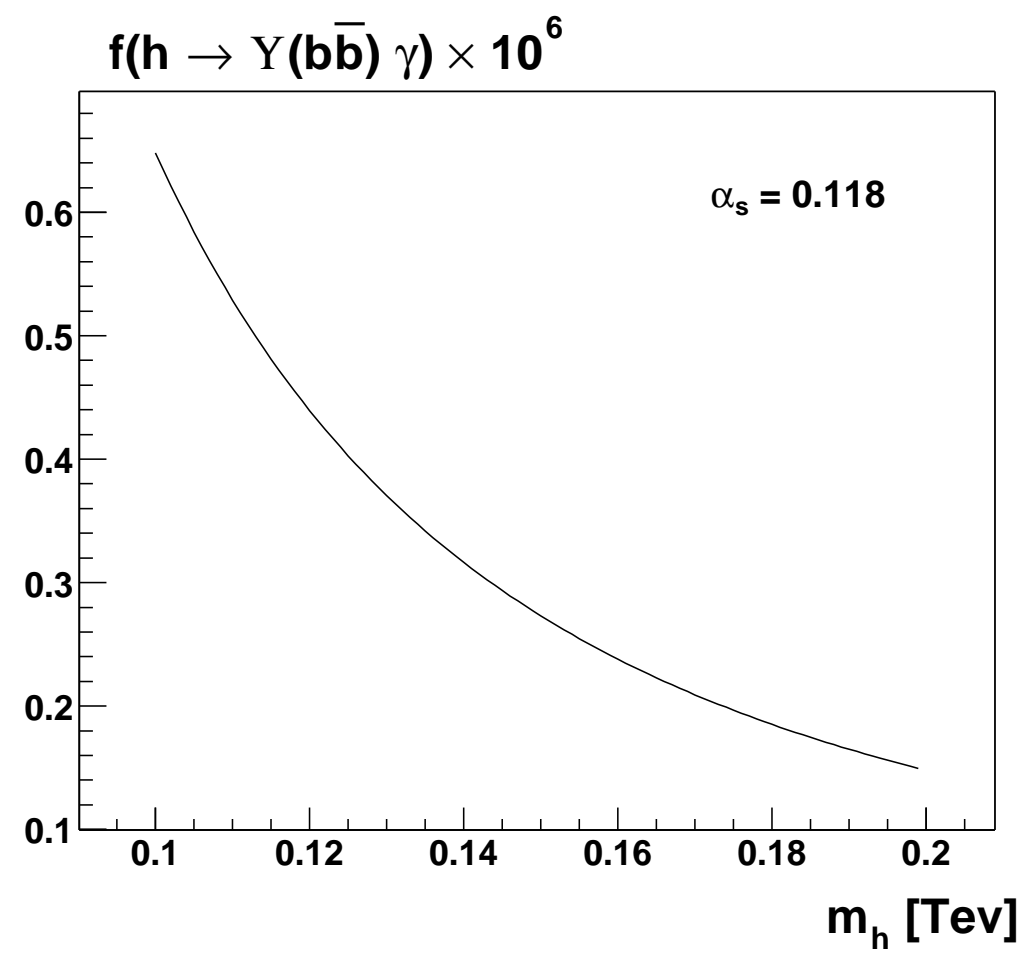

Fig.7 The relative decay width $\Gamma(h \rightarrow \Upsilon(\bar{b} b) \gamma)$ as a function of $h$-boson mass $m_{h}$ compared to $h \rightarrow \bar{b} b$ channel. 


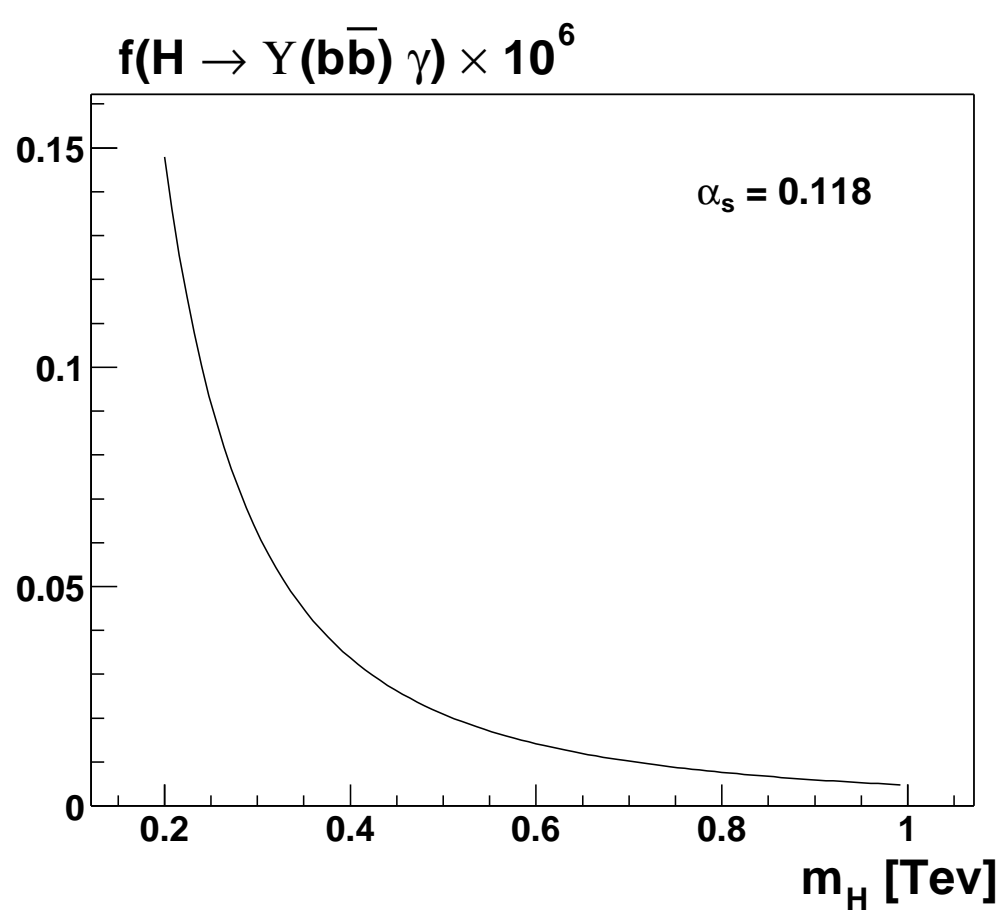

Fig.8 The relative decay width $\Gamma(H \rightarrow \Upsilon(\bar{b} b) \gamma)$ as a function of $H$-boson mass $m_{H}$ compared to $H \rightarrow \bar{b} b$ channel.

\section{EFFECTIVE MODEL FOR LOWER-ENERGY THEOREM}

Before proceeding to the lower-energy theorem applied to the transition $T \rightarrow h Z$ (Model II), we give the general expression for the amplitude of the decay mentioned above [18]

$$
\begin{aligned}
A(T & \rightarrow h Z)=\left\langle\phi_{Z}\left|\mathcal{L}_{i n t}\right| \phi_{T}\right\rangle \\
& =-\frac{1}{v}\left\langle\phi_{Z}\left|\sum_{Q=t, U, D} \frac{\alpha_{s}}{12 \pi} \eta_{h Q} G_{\mu \nu}^{a} G^{a \mu \nu}-\sum_{q=u, d, s, c, b} \eta_{h q} m_{q} \bar{q} q\right| \phi_{T}\right\rangle .
\end{aligned}
$$

Here, $\left|\phi_{Z}\right\rangle$ and $\left|\phi_{T}\right\rangle$ are eigenstates responsible for $Z$-boson and (super)heavy quarkonium $T=T(\bar{Q} Q)$, respectively. The second term in (27) corresponding to the contribution of light quarks comes directly from the interaction Lagrangian

$$
\begin{aligned}
& \mathcal{L}_{i n t}=-\frac{h}{v}\left(\sum_{l} \eta_{h l} m_{l} \bar{l} l+\sum_{q=u, d, s, c} \eta_{h q} m_{q} \bar{q} q+\sum_{Q=b, t, U, D} \eta_{h Q} m_{Q} \bar{Q} Q-\right. \\
& \left.-2 \eta_{h W} m_{W}^{2} W_{\mu}^{+} W^{\mu-}+\eta_{h Z} m_{Z}^{2} Z_{\mu}^{2}\right),
\end{aligned}
$$

while the gluonic contribution given by the operator in the first term of (27) arises from the coupling of the Higgs boson $h$ to $t$-, $U$ - and $D$-quarks through the standard loop mechanism (see, e.g., references in [18]) with $N_{h}$ number of heavy quarks in the loop. The functions $\eta_{h i}$ in $(27)$ and $(28)\left(i=\right.$ leptons $(l)$, light quarks $\left.(q), Q, W^{ \pm}, Z\right)$ are model-dependent ones describing a deviation from the SM picture where all of $\eta_{h i}$ are equal to unity. Obviously, considering the fermionic sector, only the terms containing quarks with masses $m_{Q} \geq m_{h}$ 


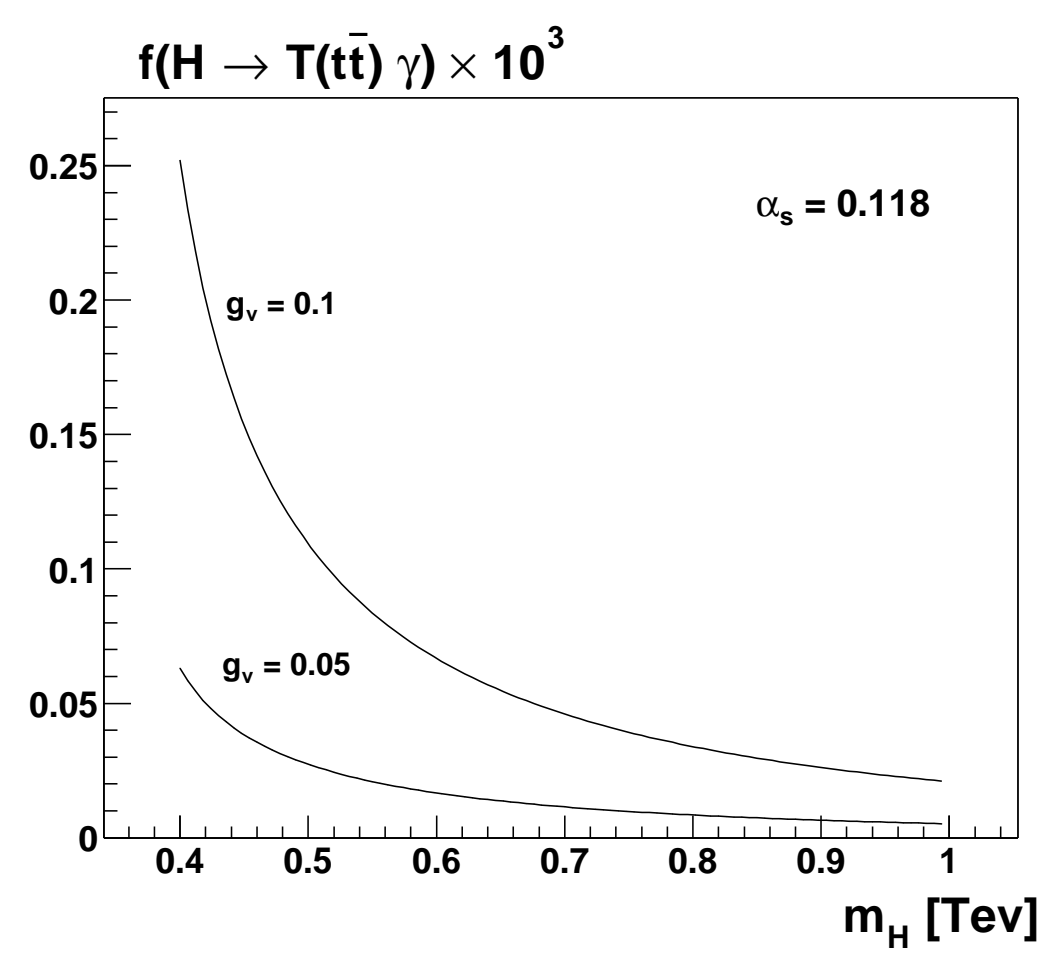

Fig.9 The relative decay width $\Gamma(H \rightarrow T(\bar{t} t) \gamma)$ as a function of $H$-boson mass $m_{H}$ compared to $H \rightarrow \bar{t} t$ channel for different values of $g_{V}$.

are relevant for giving rise to the amplitude provided by the matrix element of (27) where the only gluonic part survives. Our aim is to calculate the amplitude (27). Fortunately, QCD gives the trace of the energy-momentum tensor $\Theta_{\mu \nu}$ in the following form [18]:

$$
\Theta_{\mu \mu}=-\frac{b_{6} \alpha_{s}}{8 \pi} G_{\mu \nu}^{a} G^{\mu \nu a}+\sum_{q} m_{q} \bar{q} q+\sum_{Q} m_{Q} \bar{Q} Q,
$$

where $b_{6}$ is the first coefficient of the $\beta\left(\alpha_{s}\right)$ function in QCD with 6 flavors of quarks. At the zero momentum transfer, the matrix element of (29) between any different eigenstates $X$ and $Y$ of the Hamiltonian is vanishing $[19,18]$

$$
\left\langle X\left|\Theta_{\mu \mu}\left(q^{2}\right)\right| Y\right\rangle=0
$$

with $\left\langle X\left|\Theta_{00}\left(q_{0}^{2}, \overrightarrow{0}\right)\right| Y\right\rangle=0$. Using the condition (30), one can obtain the following relation between the matrix elements containing the gluonic part and the heavy quark terms:

$$
\left\langle\phi_{Z}\left|\frac{b_{6} \alpha_{s}}{8 \pi} G_{\mu \nu}^{a} G^{\mu \nu a}\right| \phi_{T}\right\rangle=\left\langle\phi_{Z}\left|m_{b} \bar{b} b+m_{t} \bar{t} t\right| \phi_{T}\right\rangle,
$$

where the light quark contributions are neglected. Taking into account the gluonic anomaly effect through the replacement of the top-quark mass term [19]

$$
m_{t} \bar{t} t \rightarrow-\frac{2}{3} \frac{\alpha_{s}}{8 \pi} G_{\mu \nu}^{a} G^{\mu \nu a},
$$


Eq.(31) transforms into

$$
\left\langle\phi_{Z}\left|\frac{b_{5} \alpha_{s}}{8 \pi} G_{\mu \nu}^{a} G^{\mu \nu a}\right| \phi_{T}\right\rangle=\left\langle\phi_{Z}\left|m_{b} \bar{b} b\right| \phi_{T}\right\rangle
$$

where $b_{5}=b_{6}+2 / 3$. Let us consider the amplitude $(27)$ in the following form

$$
A(T \rightarrow h Z)=-\frac{1}{v}\left\langle\phi_{Z}\left|\sum_{Q=t, U, D} \frac{\alpha_{s}}{12 \pi} \eta_{h Q} G_{\mu \nu}^{a} G^{a \mu \nu}-m_{b} \bar{b} b\right| \phi_{T}\right\rangle,
$$

where the terms containing $u, d, s$ and $c$ quarks were omitted because of its small mass effect on the intermediate quark loop. Comparing Eqs. (34) and (33), we find the amplitude for the decay $T \rightarrow h Z$ in the limit of vanishing of the four-momentum of the Higgs-boson $h$ :

$$
A(T \rightarrow h Z)=-\frac{1}{v}\left\langle\phi_{Z}\left|\frac{\alpha_{s}}{8 \pi}\left(b_{5}-\frac{2}{3} \sum_{Q=t, U, D} \eta_{h Q}\right) G_{\mu \nu}^{a} G^{a \mu \nu}\right| \phi_{T}\right\rangle,
$$

where the couplings $\eta_{h Q}$ are [16]:

$$
\begin{aligned}
& \eta_{h t(U)}=\frac{\cos \alpha}{\sin \beta}=\sin (\beta-\alpha)+\cot \beta \cos (\beta-\alpha), \\
& \eta_{h D}=-\frac{\sin \alpha}{\cos \beta}=\sin (\beta-\alpha)-\tan \beta \cos (\beta-\alpha) .
\end{aligned}
$$

For numerical estimation, we use the decoupling limit where Eqs. (36) and (37) are transformed into the following ones:

$$
\begin{aligned}
\eta_{h t(U)} & \simeq 1+z s_{2 \beta} c_{2 \beta} \tan ^{-1} \beta, \\
\eta_{h D} & \simeq 1-z s_{2 \beta} c_{2 \beta} \tan \beta,
\end{aligned}
$$

where $z=\left(m_{Z} / m_{A}\right)^{2}$. To calculate the amplitude (35), one has to estimate its matrix element as those given by the soft non-perturbative gluonic field. This contribution occurs as the excitation of the non-perturbative gluon condensate in an environment of a pair of a quark and an antiquark bound at the scale

$$
r_{\text {bound state }}^{-1} \sim \Lambda_{Q}=m_{Q} \alpha_{s}\left(\tilde{\mu} \simeq m_{Q} \alpha_{s}\right),
$$

which is larger than the scale of strong interactions $\Lambda$ with $\alpha_{s} \ll 1$. This non-perturbative effect can be estimated in the transition $T \rightarrow Z$ within a minimal point-like source $F \alpha_{s} \vec{E}^{2}(x)$ [19] with $\vec{E}$ being the electric component of the gluonic field, and an arbitrary constant $F$ defines the strength of this source. Hence, the only remaining work is to calculate the following two-point function

$$
W\left(G_{\mu \nu}\right)=i \int d x e^{i q x}\left\langle 0\left|T\left\{F \alpha_{s} \vec{E}^{2}(x), \frac{\beta\left(\alpha_{s}\right)}{4 \alpha_{s}} G_{\mu \nu}^{2}(0)\right\}\right| 0\right\rangle
$$


embedded in the non-perturbative amplitude $A_{N P}$ of the decay $T \rightarrow h Z$

$$
A_{N P}(T \rightarrow h Z)=\frac{1}{v \Lambda^{2}}\left(1-\frac{2}{3} \frac{1}{b_{5}} \sum_{Q=t, U, D} \eta_{h Q}\right) \cdot W\left(G_{\mu \nu}\right) .
$$

Here, $\beta\left(\alpha_{s}\right)=\left(-b_{5} \alpha_{s}^{2} / 2 \pi\right)+O\left(\alpha_{s}^{3}\right)$. Using the tricks followed by the authors in [12,20], the calculation of the amplitude (42) can be achieved in the framework of the QCD low-energy theorem approach in the limit $q^{2} \rightarrow 0$

$$
A_{N P}(T \rightarrow h Z)=\frac{1}{v} \frac{\pi F}{\Lambda^{2}}\left(1-\frac{2}{3 b_{5}} \sum_{Q=t, U, D} \eta_{h Q}\right)\left\langle\frac{\alpha_{s}}{\pi} G_{\mu \nu}^{2}\right\rangle_{0},
$$

where $\left\langle\left(\alpha_{s} / \pi\right) G_{\mu \nu}^{2}\right\rangle_{0}$ is the standard gluonic condensate. Using these formulas, now we can estimate the corrections due to non-perturbative fluctuations of the gluonic field in the decay $T(\bar{U} U) \rightarrow h Z$

$$
\Gamma(T(\bar{U} U) \rightarrow h Z)=\Gamma_{0}(T(\bar{U} U) \rightarrow h Z)\left(1+\delta_{N P}\right)
$$

where $\Gamma_{0}(T(\bar{U} U) \rightarrow h Z)$ is the decay width (12), while the non-perturbative correction factor $\delta_{N P}$ is defined as $\delta_{N P}=\Gamma_{N P} / \Gamma_{0}$ with

$$
\Gamma_{N P}(T(\bar{U} U) \rightarrow h Z)=\frac{1}{16 \pi m_{T}}\left|A_{N P}\right|^{2}\left(1-\frac{4 m_{h}^{2}}{m_{T}^{2}}\right)^{1 / 2} .
$$

The $\delta_{N P}$ correction is obtained at the order of the magnitude of $10^{-8}$ for the strength parameter $F=1$.

\section{CONCLUSION AND DISCUSSION}

In this paper, we have concerned ourselves with the question of existence of (super)heavy quarkonia and their decays with production of a Higgs-boson, e.g., $T(\bar{U} U) \rightarrow h Z$. The possible existence of the (super)heavy quarkonium has been studied in the framework of a simple Higgs-boson potential model (Model I) having a phenomenological "hard" Yukawa couplings $\lambda\left(m_{Q}, \xi_{\chi Q}\right)$ between the (super)heavy quark and the Higgs-boson $\chi$. Furthermore, to estimate the decay width of $T(\bar{U} U) \rightarrow h Z$ by taking into account the fluctuations of the gluonic field (Model II), we used the conformal properties of QCD. We found that the effect of non-perturbative fluctuations of the gluonic field in the total decay width $\Gamma(T(\bar{U} U) \rightarrow h Z)$ is rather small. $\Gamma_{N P}$ is quite sensitive to the strength $F$ of the gluonic point-like source, since it is proportional to square of this strength. The constant $F$ can be estimated within the approach of the Model II through the decay, e.g., $T(\bar{U} U) \rightarrow \bar{l} l(l=\mu, \tau)$ with $A_{N P} \sim\left\langle\bar{l} l\left|F \alpha_{s} \vec{E}^{2}(x)\right| 0\right\rangle$, if this decay is known. The amplitude of this process is proportional to $F$, while it is cancelled in the ratio $\Gamma(T(\bar{U} U) \rightarrow h Z) / \Gamma(T(\bar{U} U) \rightarrow \bar{l} l)$. The conformal properties of QCD are especially important in transitions between lighter hadrons, e.g. $(\bar{c} c)$ - and $(\bar{b} b)$-bound states, where the multipole expansion is more sufficient [19]. In the case of (super)heavy quarkonium transitions, the Model I yields an enhancement 
effect compared with the result coming from the Model II at $\left\langle\left(\alpha_{s} / \pi\right) G_{\mu \nu}^{2}\right\rangle_{0} \simeq 0.012 \mathrm{GeV}^{4}$. Such an enhancement of the decay width $\Gamma(T(\bar{U} U) \rightarrow h Z)$ with guaranteed criterion $c=$ $\left(\Gamma_{t o t} / \epsilon_{B}\right)<1$ could have a quite significant effect on the shapes of bound-state resonances containing (super)heavy quarks in the search for new phenomena at the Tevatron and LHC. Unfortunately, the total decay width $\Gamma_{\text {tot }}$ is unknown. In Fig.4, we show the dependence of the ratio of its total decay width $\Gamma_{\text {tot }}$ to the binding energy $\epsilon_{B}$, i.e. $c=\Gamma_{\text {tot }} / \epsilon_{B}$ on the real Yukawa couplings $\xi_{\chi Q}$ in the $2 \mathrm{HDM}$ at different values of $\chi$-boson mass. We observe that the binding critical ratio $c$ is strongly sensitive to $\xi_{\chi Q}$. Thus, we believe that in some kinematical regions there is a possibility for finding the fourth family quarks at the Tevatron's Run II (the LHC experiments will not miss it). If the scale of the Higgs-boson mass does not exceed 180 $\mathrm{GeV}$, the channels of Higgs decays to $g g, \gamma \gamma$ or $\bar{l} l\left(\mu^{+} \mu^{-}, \tau^{+} \tau^{-}\right)$may give an enhancement effect which can be interpreted as the indication of heavy quark existence. For example, the decay of the CP-even lightest Higgs-boson $h \rightarrow g g(\gamma \gamma)$ with $114 \mathrm{GeV}<m_{h}<180 \mathrm{GeV}$ could be enhanced (due to the 4th generation quark contribution) by a factor $\rho \simeq 8.9(4.5$ 5.5) for $m_{4}=200 \mathrm{GeV}$ and $\rho \simeq 8.5(4.0-5.0)$ for $m_{4}=600 \mathrm{GeV}$. On the other hand, the decays of CP-even heavy Higgs $H \rightarrow g g(\gamma \gamma)$ in the mass region $180 \mathrm{GeV}<m_{H}<800 \mathrm{GeV}$ could give $9 \leq \rho \leq 13(15 \leq \rho \leq 25)$ for $m_{4}=200 \mathrm{GeV}$ and $9 \leq \rho \leq 27(15 \leq \rho \leq 57)$ for $m_{4}=600$ $\mathrm{GeV}$. This effect on the $H$ Higgs-boson decay has a minor dependence of $\tan \beta$. We have shown that a reduction of a single $U$-quark decay width leads to a significant enhancement of the signal of the $T(\bar{U} U)$ resonance. We investigated also the possible manifestation of the CP-even Higgs-boson states $h$ and $H$ in their rare decays $h \rightarrow \Upsilon(\bar{b} b) \gamma$ and $H \rightarrow \Upsilon(\bar{b} b) \gamma$ or even the production of more heavier bound states $T(\bar{t} t)$ in the rare decays of Higgs bosons $H$. We obtain that these decays can be detectable at the forthcoming experiments at the LHC.

In the final state, the Higgs bosons $h$, the gauge bosons $Z / W^{ \pm}$and even charged Higgsbosons $H^{ \pm}$are on mass-shell. Hence, the masses of heavy quarks can be reconstructed. As a trigger, one can choose a semileptonic decays $U \rightarrow D \bar{l} \nu_{l}, U \rightarrow b \bar{l} \nu_{l}, t \rightarrow b \bar{l} \nu_{l}(l=\mu, \tau)$ and the leptonic one like $W \rightarrow l \nu_{l}$ with different lepton flavors to eliminate the backgrounds $\gamma^{*}, Z, Z^{\prime} \rightarrow \bar{l} l$. On the other hand, we suppose that the efficiency for observing $\bar{Q} Q$ events may be high enough because of the characteristic kinematics such as $U \rightarrow D W, U \rightarrow b W$ and $t \rightarrow b W$. The comparison of the measured decay width mentioned above with theoretically predicted ones can exclude or even confirm the fourth family fermions (quarks). Finally, related to our calculations here, some comments are order in the following:

a. the (super)heavy quarks are considered to be non-relativistic;

b. if the $\chi$ Higgs-boson mass is $m_{\chi} \sim \mathcal{O}(100 \mathrm{GeV})$, then the decoupling limit $\left(\xi_{\chi Q} \sim \mathcal{O}(1)\right)$ is appropriate only for $m_{Q} \geq 2 m_{t}$;

c. for the $(\bar{t} t)$-bound state with $\xi_{\chi Q} \sim O(1)$, the mass $m_{\chi}$ should be smaller than the lower bound given by the LEP 2 experiments [21];

d. if the heavy quarkonium mass is of the order $2 m_{t}$, the $\chi$-boson contribution to the combined potential (4) becomes appreciable only for large values of $\xi_{\chi t} \geq 6$.

We conclude that the (super)heavy quarkonia effects calculated here can be significant and should be considered seriously for searching for new physics beyond the SM. 


\section{ACKNOWLEDGMENTS}

It is a pleasure to thank Fabiola Gianotti, Nikolai Russakovitch and Yulian Boudagov

for helpful and stimulating discussions. We are also acknowledge M. Sher and G. Eilam for informing us of the works of Refs. [7] and [9].

[1] H.-J. He, N. Polonsky, S. Su, Phys. Rev. D 64, 053004 (2001); V.A. Novikov, L.B. Okun, A.N. Rozanov and M.I. Vysotsky, Phys. Lett. B 529, 111 (2002).

[2] N. Arkani-Hamed, A.G. Cohen and H. Georgi, Phys. Lett. B 513, 232 (2001).

[3] G.A. Kozlov, Il Nuovo Cim. A 112, 1103 (1999).

[4] G.A. Kozlov, Phys. Atom. Nucl. 63, 2293 (2000).

[5] M.J. Strassler and M. Peskin, Phys. Rev. D 43, 1500 (1991).

[6] C.S. Li, R.J. Oakes and T.C.Yuan, Phys. Rev. D 43, 3759 (1991).

[7] G. Eilam, J.L. Hewett, and A. Soni, Phys. Rev. D 44, 1473 (1991) [Erratum-ibid. D 59, 039901 (1999)].

[8] E.H. Simmons, The talk at HCP 2002, Report: BUHEP-02-39; hep-ph/0211335.

[9] M. Sher and D. Silverman, Phys. Rev. D 31, 95 (1985).

[10] H. Inazawa and T. Morii, Phys. Lett. B 203, 279 (1988); Phys. Lett. B 247, 107 (1990).

[11] K. Hagiwara et al., Nucl. Phys. B 344, 1 (1990).

[12] M.B. Voloshin, Nucl. Phys. B 154, 365 (1979).

[13] S. Kanemura and C.-P. Yuan, Phys. Lett. B 530, 188 (2002).

[14] CDF Collaboration, T. Affolder et al., Phys. Rev. D 62, 012004 (2000).

[15] D0 Collaboration, V. Abazov et al., Phys. Rev. Lett. 88, 151803 (2002).

[16] H.E. Haber, Invited talk at the 10th Int. Conf. on SUSY and Unification of Fundamental Interactions (SUSY02), 17-23 June, 2002, DESY , Report-no: SCIPP 02/38; hep-ph/0212136.

[17] M.A. Shifman and M.I. Vysotsky, Nucl. Phys. B 186, 475 (1981).

[18] A.I. Vainshtein, V.I. Zakharov and M.A. Shifman, Sov. Phys. Usp. 23, 429 (1980).

[19] M.B. Voloshin, Sov. J. Nucl. Phys. 44, 478 (1986).

[20] V.A. Novikov et al., Nucl. Phys. B 191301 (1981).

[21] The LEP Higgs Working Group , R. Bock et al., CERN-EP-2000-055 and LEP experiments, ALEPH 2000-28, DELHI 2000-050, L3-Note 2525, OPAL TN 646. 